\title{
Curiosidade, prazer e transgressão: pilares motivadores do consumo de crack
}

\section{Curiosity, pleasure, transgression: pillars of the motivation to consume crack Curiosidad, placer y transgresión: los pilares motivadores del consumo de crack}

\author{
Edna Gurgel Casanoval; Gertrudes Teixeira Lopes" ${ }^{I I}$ Lorena de Paula Gomes Carvalho"II; Amanda Gassi Muzzi ${ }^{\text {iv }}$; \\ Suelen Andrade Bernardo de Freitas'; Ana Paula Lopes Pinheiro Ribeiro ${ }^{V I}$
}

\begin{abstract}
RESUMO: Estudo descritivo, com abordagem qualitativa, cujos objetivos foram descrever as motivações que levaram os usuários a experimentarem o crack e analisar as repercussões psicossociais decorrentes dos comportamentos assumidos para manutenção do consumo. Os entrevistados foram dez dependentes de crack, sendo nove do sexo masculino e um do feminino, em um Centro de Atenção Psicossocial Álcool e Drogas (CAPS AD), localizado no Município de Duque de Caxias, na Baixada Fluminense, no Estado do Rio de Janeiro, em 2011. Os resultados evidenciaram que a curiosidade e o prazer foram decisivos na iniciação do uso do crack. As transgressões referidas nas mais variadas formas de violência caracterizam o comportamento dos usuários e a venda do corpo como forma de renda para o consumo da droga. Conclui-se que o abuso de crack pode ser um artifício capaz de transformar uma vida marcada pela falta, discriminação e ausência de perspectivas, em uma busca constante por prazer.
\end{abstract}

Palavras-Chave: Droga; curiosidade e prazer; abuso de crack; transgressão.

ABSTRACT: This qualitative, descriptive study described the motivations that led users to try crack, and analyzed the psychosocial effects of the behavior pursued to maintain consumption. The interviewees were ten crack addicts, nine male and one female, at an alcohol and drug psychosocial care center (Centro de Atenção Psicossocial Álcool e Drogas, CAPS AD) in Duque de Caxias municipality, in the Baixada Fluminense, Rio de Janeiro State, in 2011. The results showed that curiosity and pleasure were decisive in their starting to use crack. User behavior was characterized by transgressions reported in various forms of violence and the sale of the body as a source of income to support drug use. It was concluded that crack abuse can be a device for transforming a life marked by failure, discrimination and lack of prospects into a constant search for pleasure.

Keywords: Drug; crack abuse; curiosity and pleasure; transgression.

RESUMEN: Estudio descriptivo con enfoque cualitativo que tuvo como objetivos describir las motivaciones que llevaron a los usuarios a probar el crack y analizar los efectos psicosociales derivados de la conducta asumida para mantener el consumo. Los entrevistados fueron 10 adictos al crack, nueve hombres y una mujer, en un Centro de Atención Psicosocial Alcohol y Drogas (CAPS AD), situado en el municipio de Duque de Caxias, en la Baixada Fluminense, en el Estado de Río de Janeiro en 2011. Los resultados mostraron que la curiosidad y el placer fueron decisivos en el inicio del consumo de crack. Los delitos mencionados en las más variadas formas de violencia caracterizan la conducta de los usuarios caracterizan la violencia y la venta del propio cuerpo como medio de ingresos para el consumo de la droga. Se concluye que el abuso de crack puede ser un dispositivo capaz de transformar una vida marcada por la falta, la discriminación y la ausencia de perspectivas, en una constante búsqueda de placer. Palabras Clave: Droga; curiosidad y placer; abuso de crack; transgresión.

\section{INTRODUÇÃO}

A hipermodernidade, caracterizada pela fluidez, flexibilidade e indiferença aos grandes princípios estruturantes da modernidade, produziu uma mudança na relação do sujeito com o tempo presente. Sobre esta questão, iremos encontrar, frequentemente, no sujeito pós-moderno, um comportamento compulsivo do consumo de produtos. Assim, podemos entender a droga como uma mercadoria e a adição como uma forma de reação, na qual a fuga da realidade, às vezes, acontece por meio da compra compulsiva do produto-droga ${ }^{1}$.

'Enfermeira. Doutora em Enfermagem. Professora Adjunta da Faculdade de Enfermagem da Universidade do Estado do Rio de Janeiro. Tutora do Programa Educação pelo Trabalho/Rede de Atenção Psicossocial. Rio de Janeiro, Brasil. E-mail: ednagurgel@yahoo.com.br.

"Professora Titular, Faculdade de Enfermagem da Universidade do Estado do Rio de Janeiro. Pós-Doutora pela Universidade de São Paulo. Coordenadora do Grupo de Estudos e Pesquisas em Álcool e outras Drogas. Rio de Janeiro, Brasil. E-mail: gertrudeslopes@gmail.com.

플 fermeira. Pós-Graduanda em Teorias Psicanalíticas na Faculdade de Administração, Ciências, Educação e Letras. Missionária da Igreja Presbiteriana do Brasil. Rio de Janeiro, Brasil. E-mail: lorenamissoes@gmail.com.

${ }^{\mathrm{IV} G r a d u a n d a}$ de Enfermagem da Universidade do Grande Rio Professor José de Souza Herdy. Duque de Caxias, Rio de Janeiro, Brasil. E-mail: amanda.gassemuzzi@terra.com.br.

vEnfermeira do Hospital Estadual Getúlio Vargas. Rio de Janeiro, Brasil. E-mail: suellen.snb@hotmail.com.

${ }^{\mathrm{VI} G r a d u a d a}$ em Comunicação. Especialista em Propaganda e Marketing pela Fundação Getúlio Vargas. Enfermeira Residente do Hospital Universitário Pedro Ernesto/Universidade do Estado do Rio de Janeiro. Membro do Grupo de Estudos e Pesquisas em Álcool e outras Drogas. Rio de Janeiro, Brasil. E-mail: anapaulaplp@uol.com.br. 
Por outro lado, o prazer proporcionado pelo uso de substâncias psicoativas, bem como a possibilidade de viverem novas experiências também devem ser considerados quando procuramos entender o fenômeno da dependência química. Várias polêmicas acompanham o tema drogas, principalmente correlacionadas com a venda e o consumo, com a desigualdade social, a legalidade ou ilegalidade e sua criminalização ${ }^{2}$.

$\mathrm{Na}$ atualidade, existe uma supervalorização da satisfação dos desejos individuais, tornando-nos escravos do presente. Dentre os diversos efeitos que as substâncias psicoativas produzem, destaca-se o prazer imediato, o qual vem impedindo o sujeito de aprender a lidar com as decepções, que são naturais da vida² .

Entretanto, as drogas sempre estiveram presentes ao longo da história da humanidade e fez parte das experiências das sociedades em suas diferentes culturas. Assim, no final do século passado, uma droga em especial, o crack, provocou impacto na sociedade e transformou-se em grande preocupação pelos seus efeitos devastadores.

O crack chegou ao Brasil nos anos 90 e o seu uso aumentou em uma proporção exagerada. Algumas evidências apontam o início em São Paulo. Em pouco tempo, o consumo da droga atingiu outros estados da federação, sendo hoje considerado um problema de saúde pública ${ }^{3-5}$.

Esta droga, apesar de ser comercializada há pouco tempo no Rio de Janeiro, já alcança inimaginável estatística. A sua ocorrência também pode ser explicada pela rápida duração dos efeitos da substância, fazendo com que o usuário volte a utilizá-la mais vezes, e em um curto espaço de tempo, predispondo-o ao desenvolvimento da dependência em breve período.

Inicialmente, o consumo da substância atingiu a faixa de usuários atraídos pelo preço reduzido em relação à cocaína, outros em busca de efeitos mais intensos. Além disso, alguns adeptos do uso injetável de cocaína abandonaram essa via de administração com receio da contaminação pelo Vírus da Imunodeficiência Humana (HIV), escolhendo o crack como alternativa.

Além dos fatores citados anteriormente como motivadores do uso do crack, a busca por sensação de prazer também foi referida como justificativa. Este sentimento, presente inicialmente, ia progressivamente sendo substituído por um comportamento compulsivo. Este uso contínuo reduzia o usuário do crack a pensar constantemente no consumo em detrimento do sono, da alimentação, do afeto e do senso de responsabilidade ${ }^{4}$.

Quanto ao perfil sociocultural observado entre os usuários de crack, o mais recorrente corresponde ao indivíduo do sexo masculino, jovem, e adultos jovens, com baixa escolaridade, desempregados, provenientes de famílias de baixa renda ${ }^{6}$.

Além das situações anteriormente descritas, o rápido crescimento do consumo do crack, suas graves repercussões físicas, psíquicas e sociais, aumentando, principalmente, a vulnerabilidade dos grupos socialmente excluídos e de parcelas carentes da população, sustentam a escolha da temática deste estudo.

A narrativa que iremos apresentar baseia-se no pressuposto de que a dependência química do crack deve ser entendida como uma condição crônica, tratável e determinada por múltiplos aspectos da existência humana, incluindo as dimensões biológicas, psíquicas e socioculturais tanto na origem dos problemas, como nas propostas de sua abordagem? .

Diante do exposto e das consequências que o assunto suscita, recortamos como objeto da pesquisa os aspectos motivacionais para a iniciação e abuso do crack entre os dependentes atendidos em um Centro de Atenção Psicossocial Álcool e Drogas (CAPS AD).

Desse modo, frente à complexidade que o problema impõe ao usuário, à sociedade em geral e ao poder público, em seus diferentes campos, os seguintes objetivos foram traçados: descrever as motivações que levaram os usuários a experimentar o crack e analisar as repercussões psicossociais decorrentes dos comportamentos assumidos para manutenção do consumo.

\section{REVISÃo DE LiteraturA}

Historicamente, o crack surgiu nos Estados Unidos na década de 80 entre pessoas residentes em bairros pobres e marginalizados das cidades de Los Angeles, Nova York e Miami. A substância produzia uma euforia de grande dimensão e curta duração, ge rando fissura intensa e tolerância exacerbada. Por tais características, foi considerada avassaladora e passou a ser o alvo das atenções do governo e da sociedade em geral ${ }^{3}$.

Ao ser inalado, o crack chega rapidamente ao cérebro e seus efeitos são sentidos quase imediatamente, em 10 a 15 segundos; no entanto, duram em média cinco minutos, o que explica seu grande poder de causar dependência ${ }^{8}$.

Outro fator contribuinte para a dependência consiste na sensação de prazer intenso referida pelas pessoas que fazem uso da substância. Como o crack é velozmente eliminado do organismo, produzindo uma súbita interrupção da sensação de bem-estar, leva a um imenso desprazer e enorme vontade de sua reutilização?

Dentre as consequências da dependência da droga, pode-se destacar a depressão, o cansaço e a fissura, que é a compulsão para usá-la, a qual, no caso do crack, é avassaladora ${ }^{8}$.

Quanto ao padrão de consumo do crack, o comportamento compulsivo é o mais recorrente, sendo os episódios de fissura e perda de controle do uso da droga suas características primordiais, o que aumenta 
a probabilidade do uso frequente e, em consequência da dependência, em menor tempo de evolução 9 .

Diante da magnitude do problema, a busca de soluções concretas e a preocupação do governo brasileiro com o elevado uso do crack, foi instituído, entre outras iniciativas, o Plano de Enfrentamento ao Crack e outras Drogas, por meio do Decreto $\mathrm{n}^{\mathrm{0}}$ 7.179, de 20 de maio de 2010. Tal legislação tem por objetivos desenvolver um conjunto integrado de ações de prevenção, tratamento e reinserção social de usuários de crack e outras drogas, bem como enfrentar o tráfico em parceria com estados, distrito federal, municípios e sociedade civil ${ }^{10}$.

Nessa perspectiva, como parte das ações do referido Plano, realizou-se, em 2012, uma Pesquisa pela Secretaria Nacional Antidrogas (SENAD), em parceria com a Fundação Oswaldo Cruz, com pessoas residentes em 26 capitais e no distrito federal. A estimativa é de que 1.035 .000 pessoas faziam uso regular de drogas ilícitas nos últimos seis meses que antecederam a pesquisa, exceto maconha. Entre esses indivíduos, 370.000 eram usuários de crack e/ou similares, sendo $14 \%$ menores de idade - o que representa, aproximadamente, 50 mil crianças e adolescentes ${ }^{11}$. Estudo desenvolvido com objetivo de cartografar a experiência de usuário de crack em tratamento, no município de Recife/PE, evidenciou que, entre os fatores que mais influenciaram o consumo do crack pela primeira vez foi a influência dos amigos, seguido da curiosidade em saber a sensação provocada pela droga, reforçada pelo ambiente social do usuário e, também, pela sensação de pertencimento a um determinado grupo ${ }^{12}$.

\section{Metodologia}

A escolha da abordagem metodológica, cuja natureza fundamentou-se na pesquisa qualitativa descritiva, tomou como princípio a busca da subjetividade dos usuários, refletida em suas motivações e motivos para encontrarem o prazer por meio do consumo do crack, bem como as consequências psicossociais decorrentes do seu abuso.

O cenário da investigação foi um CAPS AD, localizado no Município de Duque de Caxias, na Baixada Fluminense, no Estado do Rio de Janeiro.

$\mathrm{O}$ projeto foi submetido à avaliação do Comitê de Ética e Pesquisa da Universidade do Grande Rio Prof. José de Souza Herdy, sendo aprovado sob protocolo $\mathrm{n}^{\mathrm{o}}$ 0018.0.317.000-10.

Os sujeitos do estudo foram dez usuários, dependentes de crack, sendo nove do sexo masculino e um feminino, escolhidos a partir dos seguintes critérios de inclusão: estar efetivamente registrado no CAPS AD há pelo menos seis meses e apresentar um padrão compulsivo como modalidade de uso. Os relatos foram identificados com a letra E (entrevistado) e atribuídos números sequenciais para cada usuário.

Ao iniciar as entrevistas, os sujeitos receberam esclarecimentos sobre a pesquisa e os princípios éticos que envolvem a investigação com seres humanos, de acordo com a Resolução no 196/96 do Conselho Nacional de Saúde, assinando, em seguida, o Termo de Consentimento Livre e Esclarecido ${ }^{13}$.

Os dados foram tratados de acordo com os preceitos da análise de conteúdo ${ }^{14}$. Com olhar cuidadoso para as unidades de registros, chegamos a três categorias analíticas: A curiosidade e o prazer na iniciação do uso do crack; $\mathrm{O}$ uso da transgressão para aquisição da droga; $\mathrm{O}$ corpo como moeda de troca para manutenção do uso do crack.

\section{Resultados e Discussão}

$\mathrm{Na}$ tentativa de tornar mais claro o significado atribuído pelos usuários ao consumo do crack, são apresentadas as categorias analíticas citadas anteriormente.

\section{A curiosidade e o prazer na iniciação do uso do crack}

Esta categoria dá visibilidade à influência dos usuários do crack desde a primeira experimentação, seja em um contexto de intimidade familiar (irmãos e primos), seja em um ambiente de sociabilidade externa à família (amigos, colegas e conhecidos).

A influência de familiar e amigos, bem como a curiosidade em torno dos efeitos obtidos pelo uso dessa substância constituíram-se fatores que motivaram decisivamente à primeira experimentação ${ }^{12}$.

Por outro lado, a decisão de usar (ou não) drogas surge da interação de vários e diferentes fatores complexos e menos perceptíveis, como a fragilidade da situação de rua, o vínculo familiar, os limites, o medo, as expectativas e o fascínio pelos desafios ${ }^{15}$.

Todos os respondentes admitiram que a presença de uma pessoa fazendo uso da droga instigou-os à experimentação, como resumido nos três fragmentos de depoimentos:

Porque assim, tem um primo men que é usuário. (E2)

Questões de colegas, experimentaram e mandaram eu usar e por curiosidade minha eu experimentei e na época eu gostei. (E1)

Estava na estação de Manguinhos (Rio de Janeiro) $e$ um colega estava na estação esperando o trem para vim para Saracuruna e ele estava fumando o baseado com o crack né! Aí eu usei [...]. (E3)

Como se pode perceber, os familiares e colegas aparecem como influenciadores do primeiro consumo. Para estes respondentes, o crack, inicialmente consu- 
mido como curiosidade, transformou-se em fonte de prazer. Neste aspecto, a satisfação e o prazer são sensações que não podem ser postas em termos abstratos: precisam ser subjetivamente experimentadas - vividas. Assim, a satisfação de alcançar o prazer por meio da droga é apenas momentânea, mas concreta e subjetiva ${ }^{16}$.

A necessidade de consumir mais vezes o crack está associada ao desejo de sentir mais prazer ou pode estar relacionada à tentativa de evitar os sintomas da abstinência. Assim, pesquisa realizada com usuários de crack apontou que

O sentido figurado trazido pelos usuários diante do crack nos faz perceber o quanto de prazer a substância proporciona a cada um deles e o quanto é difícil abrir mão desse prazer tão intenso e imediato. Podemos pensar no crack como paradigmático da experiência de prazer ${ }^{12: 58}$.

Entretanto, o desafio de controlar o uso da substância, frequentemente sem êxito, é um fato que acompanha o início da experimentação do crack. As pessoas até têm conhecimento de que a continuidade do uso pode levar à compulsão, mas acreditam sempre que podem controlar o consumo ${ }^{12}$.

Um carinha me ofereceu e experimentei por curiosidade. Só que perdi a vontade de ir à escola, parei de estudar. (E9)

A narrativa anterior mostra que o mundo contemporâneo convoca para o prazer e o declínio dos deveres. Assim, a busca por novas sensações ou a ideia de estar consumindo algo novo mobiliza as pessoas, reforçadas pelas novidades constantes dos apelos comerciais que se mostram atraentes e sedutores, bem como a necessidade de fazer parte de um grupo de consumidores ${ }^{17}$. Isso é o que tem potencial de mobilizar os grupos, especialmente aqueles que se encontram em vulnerabilidade social. Nessa perspectiva, usuários jovens e adultos jovens usam crack mais frequentemente associado a processos destrutivos em relação à sua vida social ${ }^{18}$.

\section{O uso da transgressão para aquisição da droga}

Uma das situações que caracterizam o comportamento dos usuários de drogas é a transgressão, que pode se evidenciar de diferentes formas, desde aquelas de menores danos pessoais até o uso de violências físicas e psicológicas.

A violência doméstica e urbana, a prática de pequenos furtos, envolvimento com assaltos e com a criminalidade são situações que, frequentemente, vulnerabilizam ainda mais o usuário de drogas, além da desigualdade de oportunidade, a pouca oferta de educação de qualidade e de profissionalização exacerbam ainda mais o estigma, a discriminação e a exclusão social ${ }^{11}$.

A seguir, evidenciam-se algumas transgressões para obter o crack, como ilustra o discurso:
Roubei as coisas de dentro de casa para vender e comprar a pedra, coisas da minha mãe, dinheiro e celular. (E6)

Como vem sendo discutido por especialistas, as primeiras transgressões acometidas pela maioria dos usuários/dependentes de drogas - e em especial do crack - são realizadas dentro do próprio lar e com pessoas a quem admiram e respeitam, como, por exemplo, a mãe. Tal prática acontece inicialmente com os familiares, mas com a progressão do uso da substância, vai se ampliando até chegar ao mundo do crime, encontrando na transgressão uma forma de conformar uma identidade ${ }^{19}$.

Assim, a correlação droga-violência demarcou uma consequência do uso abusivo de drogas, identificando o usuário como um ser violento que causa medo para a sociedade, para a família e para as pessoas que vivem no seu entorno ${ }^{20}$.

Corroborando a relação entre violência e crack, estudos mostram a venda de pertences dos familiares, assaltos, pequenos furtos, tráfico de drogas em geral e sequestros $^{21,22}$.

Eu ia para rua aplicar 171 nos outros, falando que era para passagem ou então eu vendia bala, limpava vidro. (E4)

Eu peguei um celular de uma moça que morava na comunidade. (E10)

Para estes usuários, o que vale é a promoção do prazer, independente dos riscos a que frequentemente estão expostos, para obtenção de dinheiro ou droga.

A iniciação no mundo do crime, a despeito de suas motivações e da categoria da transgressão, não evidencia conscientemente sentimentos de culpa, mas, ao contrário, se manifesta como motivo de orgulho e de autoestima ${ }^{19}$.

Eu rendia os outros no meio da rua pra poder me drogar... comecei a roubar, comecei a fazer maldade, a roubar carro para poder me drogar. (E8)

Eu já cortei cara de garota por causa de crack. (E7)

Como mostram os depoimentos mencionados, "as variações na configuração das transgressões tendem a impactar a incidência da violência em sua dinâmica e, em especial, em sua intensidade"23:74.

\section{O corpo como moeda de troca para manuten- ção do uso do crack}

A venda do corpo tornou-se uma prática corrente para aquisição do crack entre homens e, principalmente, mulheres. $\mathrm{O}$ consumo continuado exige, cada vez mais, quantidades significativas de recursos financeiros. Portanto, é no comércio do corpo que as mulheres encontram a melhor forma de obtenção de dinheiro para comprar a droga ${ }^{24}$.

Já vendi meu corpo pra comprar droga, quando eu não tinha dinheiro. (E9) 
Estudo concluiu que mulheres vendem o corpo como forma de obter renda para o consumo de crack e não se reconhecem como profissionais do sexo por praticarem sexo em troca de droga ou dinheiro para comprar a substância ${ }^{25}$.

Estudo realizado em um CAPS AD no município de Duque de Caxias, no Estado do Rio de Janeiro, identificou que, entre 113 mulheres usuárias de drogas, 69,9\% não possuíam ocupação remunerada e 12,4\% estavam envolvidas em prostituição ${ }^{26}$.

Eu já fui para o hotel com um homem, amarrei o homem na cama e roubei ele todinho, até a roupa dele. Tudo vale dinheiro! (E5)

Pode-se ressaltar, também, que essa prática tem promovido o aumento das doenças sexualmente transmissíveis entre as usuárias. Diferentemente das profissionais do sexo, a venda do corpo para obtenção do crack ou do dinheiro para comprá-lo está relacionada à fissura para aquisição da droga, o que caracteriza um sexo promíscuo, de desespero, degradação e alto risco de contaminação com as doenças sexualmente transmissíveis. As profissionais do sexo, por sua vez, utilizam medidas de proteção e cuidados em relação ao sexo seguro ${ }^{24}$. Assim, a prática de sexo inseguro faz das mulheres usuárias de crack um importante grupo de risco em relação às doenças sexualmente transmissíveis (DSTs) e à Síndrome de Imunodeficiência Adquirida (AIDS).

Acrescente-se, ainda, que, ultimamente, a maior prevalência e incidência das DSTs acontecem entre as mulheres que se submetem à prática de sexo por drogas, sem as devidas precauções para a prevenção da doença ${ }^{27}$.

Nessa transação comercial de serviços sexuais para aquisição da droga, o pagante domina a negociação, exigindo, muitas vezes, a dispensa do preservativo e a prática de modalidades de sexos inusitadas, bem como o pagamento do serviço com quantias irrisórias, levando o usuário a buscar um maior número de programas e, consequentemente, expondo-o a um maior número de parceiros para alcançar a quantia necessária para a droga. Esses ingredientes perversos, juntos, aumentam consideravelmente o risco de DSTs/ AIDS, violência e degradação 28,29 .

Como é possível perceber, o uso do crack é multifatorial e precisa ser compreendido na perspectiva de aspectos psicossociais, da vulnerabilidade dos sujeitos e da agressividade da droga.

\section{CONClusão}

O abuso de crack pode vir a ser um artifício capaz de transformar uma vida marcada pela falta de recursos, discriminação e ausência de perspectivas em uma busca constante por prazer, focado no presente, no qual os usuários tentam preencher a existência, com um objetivo concreto e factível: obter mais e mais crack.
Em relação à primeira categoria, emergente dos depoimentos, os resultados evidenciaram que a iniciação ao uso de crack teve como ponto de partida a curiosidade para experimentar a droga, movida pela socialização com familiares e amigos. A este respeito, há que se compreender que, na contemporaneidade, existe uma fluidez dos laços familiares, com os colegas e os vizinhos, predispondo a uma fragilidade nos tradicionais quadros de referências.

Quanto à segunda categoria, a transgressão, em suas diferentes modalidades e amplitudes, foi referida pelos usuários como uma maneira de obter o crack. $\mathrm{O}$ medo de ficar sem a droga, a abstinência e os efeitos psíquicos decorrentes do uso predispõem à violência, que pode ser materializada em agressões físicas, verbais, roubos, furtos e venda de pertences dos familiares.

Considerando a terceira categoria, a venda do corpo para aquisição de dinheiro ou da própria droga também foi uma prática apontada nesta pesquisa. Esse comportamento de risco apresenta-se como mais um tipo de violência a que se expõe ou que está exposto o usuário de crack. É possível que estes realizem práticas sexuais sem proteção, aumentando a possibilidade de contaminação e disseminação das DST/AIDS.

É preciso melhor compreensão dos usuários de crack mediante o fortalecimento de rede intersetorial, em todos os níveis de atenção à saúde, numa perspectiva inclusiva, de redução de danos em que se respeitem a singularidade do sujeito.

O estudo, apesar da sua grandiosidade e magnitude em termos de conhecimentos produzidos a partir dos depoimentos dos dez entrevistados sobre as motivações e motivos para a iniciação, uso e abuso do crack, não permite a generalização dos resultados. No entanto, pode produzir, por aproximação, a compreensão do fenômeno em estudos similares. Com esta limitação, sugerimos que outros estudos sejam implementados para aprofundar o conhecimento nesta área.

\section{REFERÊNCIAS}

1.Dufour CR. A arte de reduzir cabeças: sobre a nova servidão na sociedade ultraliberal. Rio de Janeiro: Companhia de Freud; 2005.

2.Acselrad GA. Educação para autonomia: construindo um discurso democrático sobre drogas. IN: Acselrad GA. Avessos do prazer: drogas, aids e direitos humanos. $2^{\mathrm{a}} \mathrm{ed}$. Rio de Janeiro: FIOCRUZ; 2005. p. 183-212.

3.Procópio A. O Brasil no mundo das drogas. Petrópolis (RJ): Vozes; 1999.

4.Nappo AS, Gauduróz JC, Raymundo M, Carlini EA. Changes in cocaine use as viewed by key informants: a qualitative study carried out in 1994 and 1999 in São Paulo. J Psychoactive Drugs. 2001; 33:241-53.

5.Centro Brasileiro de Informação sobre Drogas Psicotrópicas: I Levantamento domiciliar sobre o uso de 
drogas psicotrópicas no Brasil: estudo envolvendo as 107 maiores cidades do país. São Paulo: Universidade Federal de São Paulo; 2002.

6.Ribeiro M, Dualibi LB, Perrenoud LO, Sola V. Perfil do usuário e história natural do consumo. In: Ribeiro M, Laranjeira $\mathrm{R}$, organizadores. $\mathrm{O}$ tratamento do usuário de crack. $2^{\text {a }}$ ed. Porto Alegre (RS): Artmed; 2012. p. 39-49. 7.Cruz MS, Vargens RW, Ramôa MI. Crack: uma abordagem multidisciplinar. In: Prevenção ao uso indevido de drogas: capacitação para conselheiros e lideranças comunitárias. $4^{\mathrm{a}}$ ed. Brasília (DF): Secretaria Nacional de Políticas sobre Drogas - SENAD; 2011. p. 193-206. 8.Pacievitch T. InfoEscola [site da internet]. Crack. [citado em 13 abr de 2014] Disponível em: http://www. infoescola.com/drogas/crack.

9.Perrenoud LO, Ribeiro M. Histórico do consumo do crack no Brasil e no mundo. In: Ribeiro M, Laranjeira $\mathrm{R}$, organizadores. $\mathrm{O}$ tratamento do usuário de crack. $2^{\mathrm{a}}$ ed. Porto Alegre (RS): Artmed; 2012. p. 33-38.

10.Senado Federal(Br). Decreto n ${ }^{0} 7.179 / 2010$, de 20 de maio de 2010. Brasília(DF): Senado Federal; 2010.

11.Ministério da Saúde (Br). Secretaria Nacional de Políticas sobre Drogas. FIOCRUZ. Perfil dos usuários de crack e/ou similares no Brasil. Brasília (DF): SENAD; 2013.

12.Almeida RB, Caldas MT, Albuquerque RKR, Campos AR. Crack, a pedra lançada no SUS: desafios para uma atenção necessária. Saúde em Debate: Revista do Centro Brasileiro de Estudos de Saúde. 2013;37 (esp): 49-60.

13.Ministério da Saúde (Br). Conselho Nacional de Saúde. Diretrizes e normas regulamentadoras sobre pesquisa envolvendo seres humanos. Resolução n ${ }^{0}$ 196/96. Brasília (DF): CNS; 1996.

14.Bardin L. Análise de conteúdo. Lisboa(Pt): Edições 70; 2011.

15.Fracasso L, Torres S. Situação de rua e consumo de crack. In: Ribeiro M, Laranjeira R, organizadores. $\mathrm{O}$ tratamento do usuário de crack. $2^{\text {a }}$ ed. Porto Alegre (RS): Artmed; 2012. p. 585-97.

16.Bauman Z. Modernidade líquida . Rio de Janeiro: Jorge Zahar editor; 2001.

17.Jorge MSB, Quinderé PHD, Yassui S, Albuquerque RA. Ritual de consumo do crack: aspectos socioantropológicos e repercussões para a saúde dos usuários. Ciênc. saúde colet. 2013; 18:2909-18.
18. Ministério da Saúde (Br). Abordagens terapêuticas aos usuários de cocaína/crack no Sistema Único de Saúde. Brasilia (DF): Editora MS;2010.

19.Baptista GC. Adolescência e drogas: a escuta dos dependentes. São Paulo: Vetor; 2006.

20.Rodrigues AS, Oliveira JF, Paiva MS, Oliveira DS, Marinho MN. Representações sociais de discentes técnicos de enfermagem sobre drogas. Esc Anna Nery, 2015; 19:226-32.

21. Carvalho HB, Seibel SD. Crack cocaine use and its relationship with violence and HIV. Clinics. 2009; 64:857-66.

22.Bungay V. ,Johnson NJL, Varcoe C, Boyd S. Women's health and use of crack cocaine in context: structural and everyday violence. International Journal of Drug Policy. 2010; 21:321-9.

23.Sapori LF, Sena LL. Crack e violência urbana. In: Ribeiro M, Laranjeira R, organizadores. $O$ tratamento do usuário de crack. $2^{\mathrm{a}}$ ed. Porto Alegre (RS): Artmed; 2012. p. 74-91.

24.Nappo SA, Sanchez ZVDM, Ribeiro LA. Troca do sexo por crack. In: Ribeiro M, Laranjeira R, organizadores. $\mathrm{O}$ tratamento do usuário de crack. $2^{\mathrm{a}}$ ed. Porto Alegre (RS): Artmed; 2012. p. 566-76.

25.Zaluar A, Vallim DC, Sampaio C. Uma análise do perfil sociocultural dos usuários de crack no município do Rio de Janeiro: relatos de uma etnografia. In: Anais do II Encontro Nacional sobre a Sócio-Antropologia do Uso de Psicoativos; 2012 jul 01-02; São Paulo, Brasil. São Paulo: Associação Brasileira de Estudos Sociais do Uso de Psicoativos; 2012.

26.Silva EBO, Pereira ALF. Perfil de mulheres usuárias de cocaína e crack atendidas em um centro de atenção psicossocial. Rev enferm UERJ. 2015; 23:203-9.

27.Zanotto DF, Buchele F. Revisão sistemática sobre crack: aspectos relacionados ao uso e abuso. Saúde em Debate: Revista do Centro Brasileiro de Estudos de Saúde. 2013; 37 (esp):12-20.

28.Nappo AS, Sanches ZM, Oliveira LG. Crack, Aids and women in São Paulo, Brasil. Subst use Misuse. 2011; 46:476-85.

29.Lejuez CW, Bornovalova MA, Daughters SB, Curtin JJ. Differences in impulsitivity and sexual risck behavior among inner-city crack/cocaine users and heroin users. Drug Alcohol Depend. 2005; 77:169-75. 